\title{
Connecting the dots between ARTs and live birth outcomes
}

\section{David F. Albertini ${ }^{1}$}

Published online: 20 November 2019

(C) Springer Science+Business Media, LLC, part of Springer Nature 2019

It is indeed a long road from the initiation of an ART cycle to, in the best of worlds, delivery of a healthy child. Along the way, we as specialists in reproductive endocrinology and human embryology merge forces to tease apart the underlying pathophysiology that brings the subfertile or infertile couple to our doorstep. At the core of our efforts to treat this condition is a knowledge base derived from 50 years of study into the endocrinological underpinnings of human reproduction.

Our clinical tools in managing each case take advantage of our most recent assays offering the highest sensitivity and specificity for measuring and manipulating gonadotropins or steroids. And as each cycle reveals a pattern of ovarian steroidogenesis conducive to triggering ovulation or cancelling further action, memories are jogged back to the coordination and dynamic interplay of the lead characters-the follicle and the corpus luteum (CL). While we take for granted the upcoming egg retrieval process, what is left behind are follicular remnants that would in a fresh transfer cycle be responsible for vestigial multiple CLs functioning at some subphysiologic level during the earliest stages of embryo development and pregnancy establishment. Progesterone supplementation may save the day in getting the journey going, but what if anything is consequential in the longer term of pregnancy remains unknown.

Our issue this month examines two important parameters of reproductive physiology in the context of current ART practices. First, we will consider the problem of premature luteinization (PL)-the natural outcome of ovarian stimulation when follicle intrinsic mechanisms limiting spontaneous differentiation become compromised in the periovulatory period. While the factors preventing luteinization seem to reside in the oocyte, exactly how ovarian stimulation perturbs the delicate balance of forces restraining transformation of the Graafian follicle into the CL remains obscure. Hussein and

David F. Albertini

eicjarg@gmail.com

1 Center for Human Reproduction, New York, NY, USA collaborators review the clinical manifestations of PL and the available management strategies used by clinicians (https://doi.org/10.1007/s10815-019-01598; Revisiting debates of premature luteinization and its effect on assisted reproductive technology outcome).

Over and above the more immediate issues associated with perturbations of the follicle-CL dialogue in the earliest phases of an ART cycle, conversations of quite another kind are taking place between the conceptus and soma, more specifically with the CL. The essential physiological intermediate in this conversation is of course human chorionic gonadotropin (hCG) becoming a vital measure of trophectoderm performance during the critical phase of implantation and placentation. But further on down on the road to a term gestation comes the matter of what the consequence of subphysiologic CL function means to the developing fetus and maternal health. As first suggested by Conrad and Baker in 2013 [1], their work and that of others continue to unravel what appears to be effects on the vascular health of women who have undergone ART to become pregnant [2]. They have linked both the absence or presence of excess CLs to adjustments in maternal vascular physiology [3] in early pregnancy, and report the link of this condition to preeclampsia risk [4]. The hemodynamics of pregnancy have long been understood to be a complicated and demanding requirement for the duration of pregnancy, and knowing more about these adjustments in the context ART cycles seems an imperative given the above findings.

Even more disconcerting are the responses evident within the reproductive tract requiring interventions to initiate and sustain the interactions between the conceptus, the CL, and endometrium. Here for example comes new knowledge about the critical and local role of the CL in angiogenesis and inflammation associated with normal aspects of periovulatory physiology [5]. This situation appears coupled with the recently identified role of 2-methoxyestradiol as a modulator of function by decreasing angiogenesis without affecting the ability of hCG to stimulate steroidogenesis. Updating our approaches to attend to subtle matters of implantation biology mentioned here and elsewhere seems fully warranted and 
speaks to adjustments being made in FET with respect to artificial or natural cycles of embryo transfer [6].

The second issue we draw attention to this month pertains to hCG. We urge our readers to take note of the article by $\mathrm{Al}$ Mammari and colleagues from McGill University who put into context what could and should be the most direct measure of embryo output and chances of pregnancy establishment (https://doi.org/10.1007/s10815-019-01583 Revisiting serum $\beta$-hCG cut-off levels and pregnancy outcomes using single embryo transfer).

In setting new cutoff levels for monitoring hCG output following single embryo transfer (SET), we are beginning to get a sense for just how efficient the developing trophoblast and its derivatives are at establishing and maintaining hCG output. While we assume that this critical component of the conversation noted above is NOT substantively influenced by ART procedures, like assisted zona hatching or trophectoderm biopsy, any disturbance in hCG secretion is likely to compromise pregnancy chances. With environmental factors entering the formula for pregnancy success at the level of hCG productivity [7], the time has come to scrutinize practices now embedded in routine ART care with respect to the delicate balance of interactions between the collective targets involved in implantation and beyond [8].

More than a matter of convenience or timeliness, connecting the dots between the steps of an ART cycle remains a challenging exercise for practitioners and patients alike. For one thing, it is a moving target: tests are refined and revised as assays needing updating (the classic example is $\mathrm{AMH}$ ), addons come in and out of clinical circles waiting for a clear answer, and boiler plate treatment strategies just do not work. Collectively we look forward to a time of solidarity and uniformity, achievable perhaps, once the underlying principles of human reproduction gain access to the realm of clinical ART.

\section{References}

1. Conrad KP, Baker VL. Corpus luteal contribution to maternal pregnancy physiology and outcomes in assisted reproductive technologies. Am J Physiol Regul Integr Comp Physiol. 2013;304(2):R6972.

2. Conrad KP, Petersen JW, Chi YY, Zhai X, Li M, Chiu KH, et al. Maternal cardiovascular dysregulation during early pregnancy after in vitro fertilization cycles in the absence of a corpus luteum. Hypertension. 2019;74(3):705-15.

3. von Versen-Hoynck F, Narasimhan P, Selamet Tierney ES, Martinez N, Conrad KP, Baker VL, et al. Absent or excessive corpus luteum number is associated with altered maternal vascular health in early pregnancy. Hypertension. 2019;73(3):680-90.

4. von Versen-Hoynck F, Schaub AM, Chi YY, Chiu KH, Liu J, Lingis $\mathrm{M}$, et al. Increased preeclampsia risk and reduced aortic compliance with in vitro fertilization cycles in the absence of a corpus luteum. Hypertension. 2019;73(3):640-9.

5. Lu E, Li C, Wang J, Zhang C. Inflammation and angiogenesis in the corpus luteum. J Obstet Gynaecol Res. 2019.

6. Henriquez S, Kohen P, Xu X, Veenstra TD, Munoz A, Palomino WA, et al. Oestrogen metabolites in human corpus luteum physiology: differential effects on angiogenic activity. Fertil Steril. 2016;106(1): 230-7 e1.

7. Paulesu L, Rao CV, Ietta F, Pietropolli A, Ticconi C. hCG and its disruption by environmental contaminants during human pregnancy. Int J Mol Sci. 2018;19(3).

8. Hobeika E, Singh S, Malik S, Knochenhauer ES, Traub ML. Initial maternal serum human chorionic gonadotropin levels in pregnancies achieved after assisted reproductive technology are higher after preimplantation genetic screening and after frozen embryo transfer: a retrospective cohort. J Assist Reprod Genet. 2017;34(10):1333-40.

Publisher's note Springer Nature remains neutral with regard to jurisdictional claims in published maps and institutional affiliations. 